\title{
Evaluation of the effects of cross-linking and swelling on the mechanical behaviors of hydrogels using the digital image correlation method
}

Makoto Uchida, Tatsuya Sengoku, Yoshihisa Kaneko, Dai Okumura, Hiro Tanaka and Shohei Ida

\begin{tabular}{|c|l|}
\hline Citation & Soft Matter, 15(16); 3389-3396 \\
\hline Issue Date & $2019-04-28$ \\
\hline Type & Journal Article \\
\hline Textversion & Author \\
\hline Relation & $\begin{array}{l}\text { The following article has been accepted by Soft Matter. This is not the published } \\
\text { version. The final, published version is available at } \\
\text { https://doi.org/10.1039/c9sm00049f }\end{array}$ \\
\hline DOI & \begin{tabular}{l}
$10.1039 / \mathrm{C} 9 \mathrm{SM} 00049 \mathrm{~F}$ \\
\hline
\end{tabular} \\
\hline
\end{tabular}

Self-Archiving by Author(s)

Placed on: Osaka City University Repository

https://disv03.media.osaka-cu.ac.jp/il/meta_pub/G0000438repository

UCHIDA, M., SENGOKU, T., KANEKO, Y., OKUMURA, D., TANAKA, H., \& IDA, S. (2019). Evaluation of the effects of cross-linking and swelling on the mechanical behaviors of hydrogels using the digital image correlation method. Soft Matter. 15, 3389-3396. 


\title{
Evaluation of effects of cross-link and swelling on mechanical behavior of hydrogel using digital image correlation method
}

\author{
Makoto Uchida $^{* a}$, Tatsuya Sengokua ${ }^{a}$ Yoshihisa Kaneko ${ }^{a}$, Dai Okumurab ${ }^{b}$, \\ Hiro Tanaka ${ }^{\mathrm{c}}$ and Shohei Ida ${ }^{\mathrm{d}}$ \\ ${ }^{a}$ Graduate School of Engineering, Osaka city university, 3-3-138, Sugimoto, Sumiyoshi-ku, Osaka, 558-8585, Japan, Email: uchida@osaka-cu.ac.jp \\ ${ }^{\mathrm{b}}$ Graduate School of Engineering, Nagoya University, Furo-cho, Chikusa-ku, Nagoya, 464-8601, Japan \\ ${ }^{\mathrm{c}}$ Graduate School of Engineering, Osaka University, 1-1 Yamadaoka, Suita, Osaka 565-0871, Japan \\ ${ }^{d}$ Faculty of Engineering, The University of Shiga Prefecture, 2500, Hassaka-cho, Hikone-City, Shiga 522-8533 Japan Materials Science
}

\begin{abstract}
Experimental evaluation and modeling are important steps in the investigation of mechanical behaviors of hydrogels in the small- to large-strain range. In this study, the effects of cross-link and swelling on the true stress-strain response of a specific type of hydrogel (polyacrylamide) were evaluated using a uniaxial tensile test. The development of true strain on the surface of the hydrogel was measured using the digital image correlation method. The specimens with higher cross-link density exhibited a higher initial elastic modulus and earlier orientation hardening. The initial elastic modulus was reduced by the swelling; whereas, the orientation hardening occurred at an earlier strain range in the swollen hydrogel. The mechanical responses of the as-prepared and swollen hydrogel with different values of cross-linker contents were fitted using the non-Gaussian statistical model. The conventional model underestimated the decrease in the elasticity owing to the swelling effect and overestimated the increase in the stress in the large-strain range. The mechanical model was suitably modified to yield an accurate reproduction of the mechanical responses. The proposed model, which was characterized by five material parameters, was found to reproduce the characteristics of the mechanical response of the as-prepared and swollen hydrogels with different values of cross-linker contents.
\end{abstract}

Keywords: Hydrogel, Swelling, True stress-true strain, Digital image correlation, non-Gaussian statistic model, Rubber elasticity.

\section{INTRODUCTION}

Hydrogels are soft polymer materials that hold solvents in the three-dimensional structure of their molecular chain network. They are employed in a wide range of biomedical applications, such as contact lenses, corneal implants, substitutes for ligaments, and cartilage. The mechanical behavior of the material strongly depends on the cross-link density and degree of swelling. Research studies [16] have established coupled models of mechanics-chemistry for predicting of the changes in the mechanical properties during the swelling process. To describe the swelling process in elastomers, a free-energy function (estimated from the mechanical stretching and the mixing of polymer and solvent molecules) was introduced in the Flory-Rehner model [1]. Subsequently, a non-Gaussian statistic was incorporated in the model to describe the hardening of the material owing to the orientation of the 
molecular chain in the large-strain range [2, 3]. Meanwhile, Okumura et al. [4, 5] introduced two scaling exponents to estimate the strain energy functions separated into deviatoric and volumetric parts. Their model successfully reproduced the effect of swelling on Young's modulus for several combinations of elastomer and solvent. Recently, they predicted the ultimate swelling characterized by the limiting chain extensibility using the non-Gaussian models [6].

A mechanical test for the gel material is required to validate the employed model and evaluate the parameters used in the model. The effect of the cross-link density and swelling on the initial stiffness is accurately evaluated by the respective tests for tension, compression, and shear oscillation [7-12]. In these studies, polyacrylamide hydrogel was generally employed as a model material to understand the swelling process [9-12]. Polyacrylamide hydrogel has a tendency to deform until an excessively high stretch ratio. Therefore, the experimental evaluation and modeling of the stress-strain relationship of the material from the small- to large-strain range are of utmost importance. Recently, several optical methods for measuring strain in hydrogels have been investigated [13-16]. Digital image correlation (DIC) is widely established as highly effective and accurate optical method for measuring the strain on a specimen surface. Uchida and Tada [17] proposed the netDIC method to evaluate the strain development during the neck propagation process in thermoplastic polymers [17], the macroscopic strain concentration of coarse-grained pure-copper specimens [18], and the microscopic non-uniform deformation of polycrystalline structures [19]. It enables the evaluation of the displacement field on a specimen surface until the significantly large-strain range.

In this study, the effects of cross-link and swelling on the true stress-strain relationship of the hydrogel from the small- to the large-strain range were experimentally evaluated by performing the uniaxial tensile test on polyacrylamide hydrogel specimens, with different degrees of cross-linker contents and swelling. To measure the true strain of the specimen accurately during the tensile test, the development of the displacement field of the specimen was evaluated using the netDIC. Eventually, the mechanical responses of the as-prepared and swollen specimens of hydrogel with different crosslinker contents were fitted using the non-Gaussian statistic model.

\section{EXPERIMENTAL METHOD}

2.1 Preparation of hydrogel specimen

The polyacrylamide hydrogel employed in this study has high water content held in its microscopic, three-dimensional structure of molecular chain network, which is composed of acrylamide (AAm) and $N, N^{\prime}$-methylenebisacrylamide (BIS). As the first step in the preparation of the specimen, AAm and BIS, which are a monomer and a cross-linker, respectively, were dissolved in water. To evaluate the effect of the cross-link density on the stress-strain relationship of the hydrogel, specimens with different BIS content were prepared, as illustrated in Table 1. The specimens prepared using BIS contents of 2, 5, and $10 \mathrm{mM}$ were referred to as BIS2, BIS5, and BIS10, respectively; 
moreover, the BIS content is denoted by $\beta$ ( $\beta=2,5$, and10 for BIS2, BIS5 and BIS10, respectively). Subsequently, polymerization was conducted using ammonium persulfate (APS) and tetramethylethylenediamine (TMEDA), which are the initiator and accelerator, respectively. Specimens of dimensions 30 (length) $\times 10$ (width) $\times 1$ (thickness) $\mathrm{mm}$ were formed using silicone molds. The effect of swelling on the mechanical behavior of the hydrogel was also investigated. In this study, as-prepared and fully swollen specimens (referred to as Specimens AP and SW) were prepared. All the conditions employed in this study are listed in Table 2. The volume increase ratios were evaluated by measuring the weight of the gel specimen.

Table 1 Component of polyacrylamide hydrogel

\begin{tabular}{cccccc}
\hline & AAm $(\mathrm{g})$ & BIS $(\mathrm{mg})$ & APS $(\mathrm{mg})$ & TMEDA $(\mathrm{mL})$ & $\mathrm{H}_{2} \mathrm{O}(\mathrm{mL})$ \\
\hline Specimen-BIS2 & 3.73 & 4.6 & 17.1 & 0.0449 & 14.95 \\
Specimen-BIS5 & 3.73 & 11.6 & 17.1 & 0.0449 & 14.95 \\
Specimen-BIS10 & 3.73 & 23.1 & 17.1 & 0.0449 & 14.95 \\
\hline
\end{tabular}

Table 2 Characteristics of hydrogel specimens

\begin{tabular}{|c|c|c|c|c|}
\hline & $\beta \quad[\mathrm{mM}]$ & Swelling time & Volume increase ratio & Sample number \\
\hline BIS2-AP & 2 & As prepared & 1.0 & 5 \\
\hline BIS2-SW & 2 & $24 \mathrm{~h}$ & $3.32 \pm 0.07$ & 5 \\
\hline BIS5-AP & 5 & As prepared & 1.0 & 5 \\
\hline BIS5-SW & 5 & $24 \mathrm{~h}$ & $2.56 \pm 0.15$ & 5 \\
\hline BIS10-AP & 10 & As prepared & 1.0 & 5 \\
\hline BIS10-SW & 10 & $24 \mathrm{~h}$ & $2.27 \pm 0.01$ & 5 \\
\hline
\end{tabular}

\subsection{Tensile test}

As the tensile force acting on the hydrogel specimen was small, a load cell of high sensitivity was required for the test. We used a precision electronic balance to capture the tensile load accurately, as shown in Fig. 1 (a). The lower side of the hydrogel specimen was held by the lower chuck to which a weight of approximately 1,100 gf was attached; meanwhile, the upper side was held by the upper chuck that in turn was connected to the movable crosshead. The reading on the balance was reset to zero when the tensile load was not applied to the specimen. When the tensile force was applied to the specimen through the crosshead movement, the balance reading displayed a negative value because the force was acting in the upward direction through the weight and specimen. The tensile test was performed at a constant rate of tensile displacement of $5 \mathrm{~mm} / \mathrm{min}$ (which corresponded to a nominal strain rate of approximately $5 \times 10^{-3} / \mathrm{s}$ ), whereas the tensile load was recorded by the computer. The test was performed three times for each specimen listed in Table 2. 


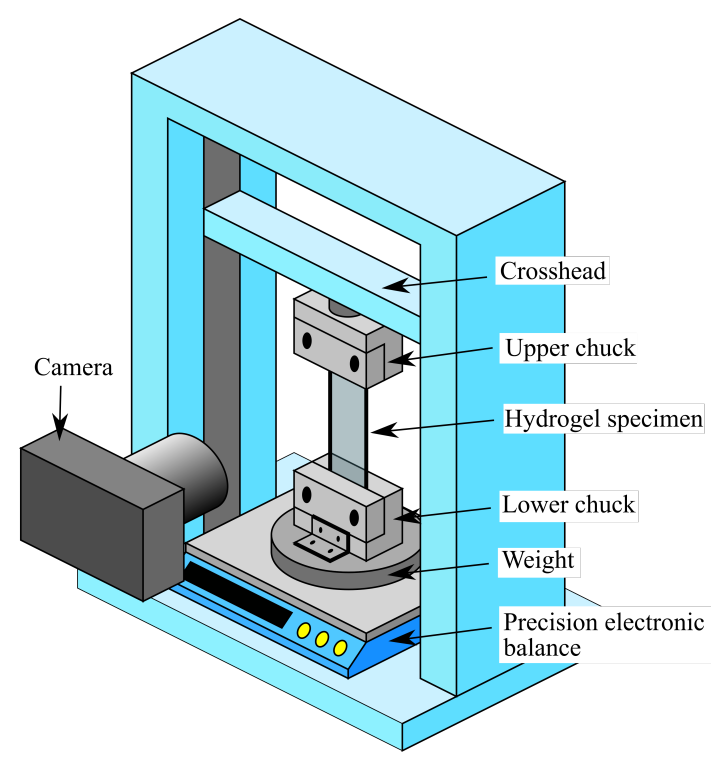

(a) Setup for tensile test



(b) Preparation of DIC

Fig. 1 Experimental method for uniaxial tensile test of hydrogel

\subsection{Evaluation of true strain and stress}

Digital image correlation is a contactless, full-field, two-dimensional measurement method; here the displacement of the point-of-interest (POI) is identified using the brightness distribution on the subset configured around the POIs on two images. When a large and/or non-uniform deformation occurs between two images, the shape change in the subset as well as the displacement of the POI should be identified. In a standard DIC, the function for the displacement field to represent the shape change in the subset is generally evaluated by the iterative scheme; which requires substantial computational time and occasionally diverges owing to the complex nonuniform deformation and/or a large deformation caused between the images. In this study, we employed the netDIC [17] to achieve the strain evaluation until the significantly large-strain range. In the netDIC, the network was constructed using the measurement points, and the displacements of the points were nonlocally evaluated by introducing the shape change in the subset based on the deformation of the network. Because the shape change in the subset can be introduced explicitly in the netDIC, the higher-order equation can be employed to describe the shape change in the subset without additional computational time. It enables an accurate and stable measurement even when a large and/or non-uniform deformation occurs between the images.

For the measurement by DIC, a brightness distribution has to be provided on the surface of the object. The random speckle pattern is frequently provided by spray. In this study, the dot-array pattern was employed for the brightness distribution; it was provided on the surface of the hydrogel specimen using a watercolor ink pen, as shown in Fig. 1 (b). About 48 to 60 POIs were allocated in the vicinity 
of the dots. The digital image of the specimen surface was captured using a digital single-lens reflex camera (Body: K20, Pentax Co. Ltd., Lens: DFA Macro 50 mm F2.8, Pentax Co. Ltd.) every 15 s. The spatial resolution of the digital image was approximately $45 \mu \mathrm{m} /$ pixel.

After obtaining the displacements of the measurement points, the strain field was evaluated. The local true strain tensor was calculated from the deformation gradient tensor of the local triangular element constructed using the points whose displacements had been measured. The global true strain $\bar{\varepsilon}_{22}$ and global true stress $\bar{\sigma}_{22}$ in the tensile direction were then evaluated as

$$
\begin{aligned}
& \bar{\varepsilon}_{22}=\frac{1}{S} \int_{S} \varepsilon_{22} d S \\
& \bar{\sigma}_{22}=\frac{F}{A_{0}} \exp \left(\bar{\varepsilon}_{22}\right)
\end{aligned}
$$

where $S$ is the area of the displacement evaluation region, $F$ is the tensile load, and $A_{0}$ is the initial cross section of the hydrogel specimen.

\subsection{Mechanical model}

The mechanical behavior of a polymeric material at temperatures above the glass transition temperature exhibits the characteristics of rubber elasticity. Arruda and Boyce [20] proposed the nonGaussian statistic model to describe the three-dimensional rubber elastic response. In this model, the initial length of a molecular chain is assumed as $\sqrt{N} l_{0}$; here, $N$ is the number of segments, and $l_{0}$ is the length of a segment. Therefore, the critical stretch $\lambda_{\max }=N l_{0} / \sqrt{N} l_{0}=\sqrt{N}$ is introduced into the stress-strain response in this model. Here, we assumed that the reference state of the molecular chain corresponds to the as-prepared hydrogel. When the volume changes by swelling or de-swelling, the molecular chain stretches or shrinks. It changes the capacity for mechanical stretch of the swollen/de-swollen hydrogels. The relationships between Cauchy stress $\sigma$ and the left CauchyGreen stretch tensor $\boldsymbol{V}^{2}=\boldsymbol{F} \cdot \boldsymbol{F}^{T}$ is expressed as

$$
\boldsymbol{\sigma}=\frac{1}{3} C_{R} \sqrt{N} \frac{\boldsymbol{V}^{2}}{\lambda_{c}} \mathcal{L}^{-1}\left(\frac{\lambda_{c}}{\sqrt{N}}\right)
$$

where $C_{R}=n k_{B} T$ is the rubber elastic modulus, $n$ is the molecular chain density, $\lambda_{c}=\left\{\operatorname{tr}\left(\boldsymbol{V}^{2}\right) / 3\right\}^{1 / 2}$ is the molecular chain averaged stretch, and $\mathcal{L}^{-1}$ is the inverse function of the Langevin function $\mathcal{L}(x)=\operatorname{coth} x-1 / x$. The material constants in this model are $C_{R}$ and $\sqrt{N}$, which correspond to the initial slope and limit stretch of the stress-strain curve, respectively. These constants are defined at the stress-free crosslinking state. 


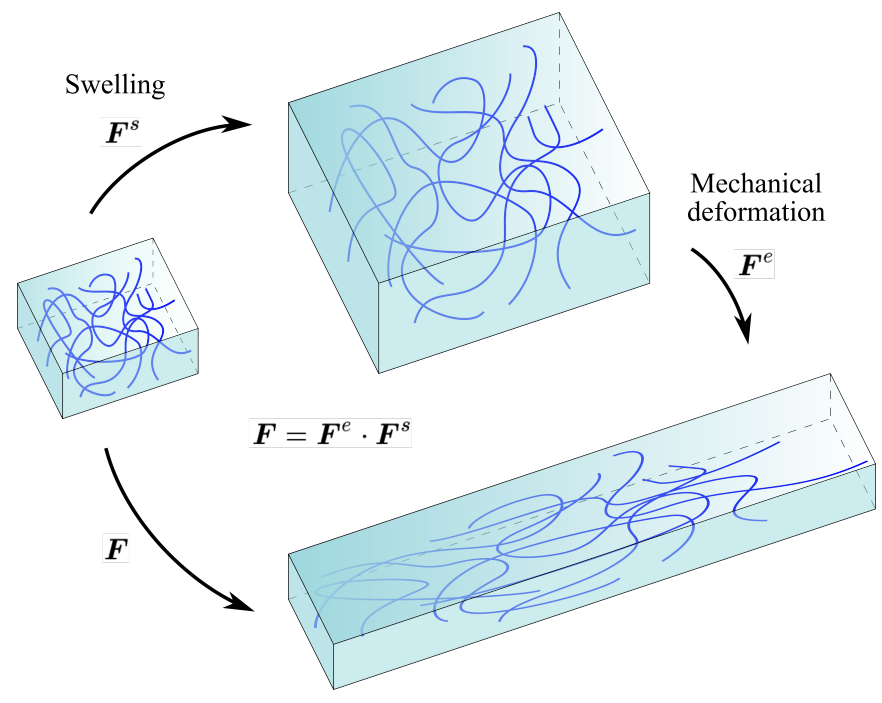

Fig. 2 Schematic of decomposition of deformation gradient.

The total deformation gradient was assumed to be decomposed by the mechanical part $\boldsymbol{F}^{e}$ and swelling part $\boldsymbol{F}^{s}$, as shown in Fig. $2[2,3]$

$$
\boldsymbol{F}=\boldsymbol{F}^{e} \cdot \boldsymbol{F}^{s} .
$$

Here, we considered the isotropic swelling process. In this case, $\boldsymbol{F}^{s}$ is expressed as

$$
\boldsymbol{F}^{s}=\lambda^{s} \boldsymbol{I},
$$

where $\lambda^{s}$ is the isotropic swelling stretch. The left Cauchy-Green stretch tensor is then re-expressed as

$$
\boldsymbol{V}^{2}=\boldsymbol{F} \cdot \boldsymbol{F}^{T}=\boldsymbol{F}^{e} \cdot \boldsymbol{F}^{s} \cdot\left(\boldsymbol{F}^{e} \cdot \boldsymbol{F}^{s}\right)^{T}=\left(\lambda^{s}\right)^{2} \boldsymbol{F}^{e} \cdot\left(\boldsymbol{F}^{e}\right)^{T}=\left(\lambda^{s}\right)^{2}\left(\boldsymbol{V}^{e}\right)^{2}
$$

The mechanical and swelling chain stretches, $\lambda_{c}^{e}$ and $\lambda_{c}^{s}$, which relate to $\boldsymbol{F}^{e}$ and $\boldsymbol{F}^{s}$, respectively, are expressed as

$$
\begin{aligned}
& \lambda_{c}^{e}=\sqrt{\frac{1}{3} \operatorname{tr}\left\{\left(\boldsymbol{V}^{e}\right)^{2}\right\}}, \\
& \lambda_{c}^{s}=\sqrt{\frac{1}{3} \operatorname{tr}\left\{\left(\boldsymbol{V}^{s}\right)^{2}\right\}}=\lambda^{s} .
\end{aligned}
$$

For the isotropic swelling case, the chain stretch becomes simplified as 


$$
\lambda_{c}=\sqrt{\frac{1}{3} \operatorname{tr}\left(\boldsymbol{V}^{2}\right)}=\sqrt{\frac{1}{3} \operatorname{tr}\left\{\left(\boldsymbol{V}^{e}\right)^{2}\right\}\left(\lambda^{s}\right)^{2}}=\lambda_{c}^{e} \lambda^{s}
$$

Because the density of the molecular chain $n$ is changed by the swelling, the rubber elastic modulus $C_{R S}$, which is proportional to $n$, is expressed as

$$
C_{R S}=C_{R}\left(\lambda^{s}\right)^{-3}
$$

Therefore, the true stress after swelling is expressed as

$$
\boldsymbol{\sigma}=\frac{1}{3} C_{R} \sqrt{N}\left(\lambda^{s}\right)^{-2} \frac{\left(\boldsymbol{V}^{e}\right)^{2}}{\lambda_{c}^{e}} \mathcal{L}^{-1}\left(\frac{\lambda^{s} \lambda_{c}^{e}}{\sqrt{N}}\right)
$$

The term $\mathcal{L}^{-1}\left(\lambda^{s} \lambda_{c}^{e} / \sqrt{N}\right)$ is re-expressed as $\mathcal{L}^{-1}\left(\lambda_{c}^{e} / \sqrt{N^{\prime}}\right)$ with $\sqrt{N^{\prime}}=\sqrt{N} / \lambda^{s}$. This indicates the decrease in the limit of the mechanical stretch owing to the stretch by swelling. Hereafter, this mechanical model is referred to as the AB model. It may be noted that Eq. (11) is not a coupled model of mechanics-chemistry. Although this equation represents the mechanical behavior of a swollen hydrogel, it does not facilitate the prediction of the swelling process. In this study, the mechanical stretch, $\lambda^{e}$, was measured by DIC; moreover, the isotropic stretch by swelling, $\lambda^{s}$, was estimated from the volume increase presented in Table 2.

\section{EXPERIMENTAL RESULTS}

The tensile tests were performed using the hydrogel specimens listed in Table 2. The strain

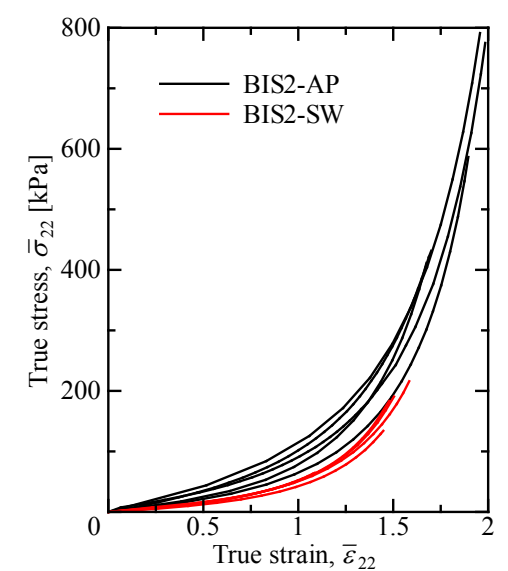

(a) BIS2

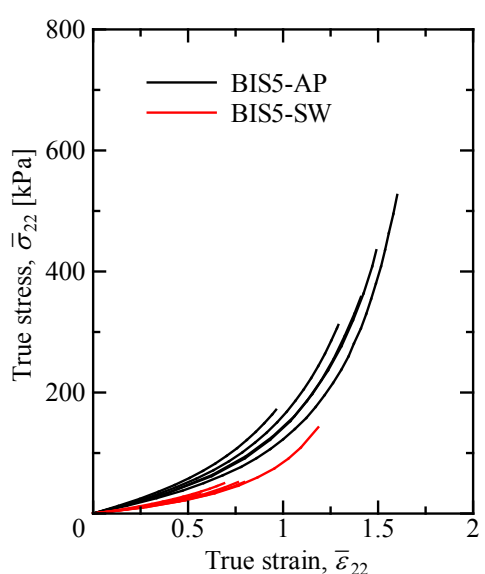

(b) BIS5

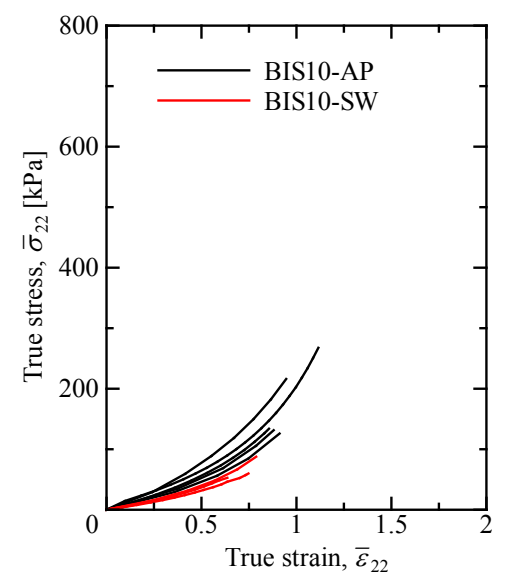

(c) BIS10

Fig. 3 Relationships between true stress and true strain (Five results are presented for each specimen). 
distribution on the surface of the specimen was evaluated using the netDIC during the tensile test. The relationships between true stress and true strain were evaluated using Eqs. (1) and (2). The responses obtained for the specimens BIS2, 5, and 10 are shown in Figs. 3 (a), (b), and (c), respectively. The results for the five uniaxial tensile tests are shown in respective specimens. The true stress-strain curve demonstrates that the response is similar to the general rubber elasticity accompanied by the orientation hardening of the molecular chain in the large-strain range. A larger variability was observed for specimens AP, whereas the responses are almost similar for specimens SW. This variability is likely to be related to the deviation in the strength of specimens AP. Meanwhile, this deviation is moderated for specimens SW owing to the equilibrium swelling state.

The hydrogel specimens with the larger cross-link density display higher initial elastic modulus and earlier fracture. A decrease in the initial elastic modulus by an increase in the swelling was observed, for all the BIS conditions. This reduction by means of swelling is attributable to the reduction in the molecular chain density of the specimen. Furthermore, in the case of the swollen hydrogel, the orientation hardening occurred at an earlier strain range. This result indicates that the solvent present in the polymer network caused the extension of the molecular chain, thereby resulting in the decrease in the extensibility. A similar result was obtained from the mechanical simulation of a swollen hydrogel by using the non-Gaussian model considering limiting chain extensibility [6].

The true stress-strain curves for the specimens with different $\beta$ were fitted using the $\mathrm{AB}$ model. The curve fitting using the material parameters was based on the nonlinear least square method,

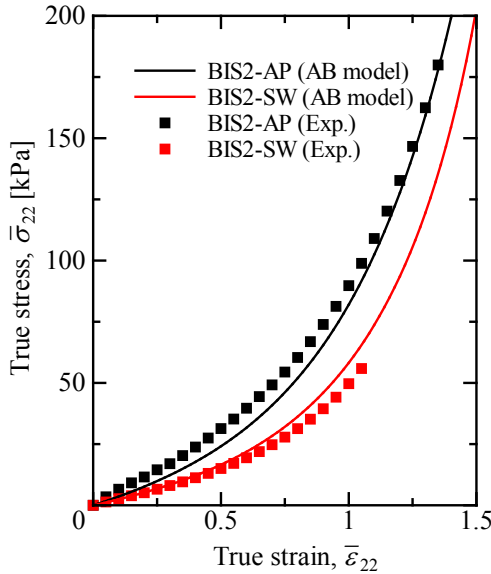

(a) BIS2



(b) BIS5



(c) BIS 10

Fig. 4 Relationships between true stress and true strain fitted by AB model.

Table 3 Fitted parameters in AB model for each specimen

\begin{tabular}{cccc}
\hline & Specimen-BIS2 & Specimen-BIS5 & Specimen-BIS10 \\
\hline$C_{R}[\mathrm{kPa}]$ & 11.1 & 19.8 & 25.1 \\
$N$ & 35.5 & 20.1 & 14.3 \\
\hline
\end{tabular}


with numerical differentiation of the stress for each parameter. The results obtained and the parameters fitted for each specimen are presented in Fig. 4 and Table 3, respectively. The experimental results plotted by the square marker are the averaged responses of curves for all the condition shown in Fig. 3. The $\mathrm{AB}$ model is found to effectively describe the non-linear mechanical response and the decrease in the rubber elasticity owing to the swelling. However, a larger decrease in the initial rubber elasticity for the increased swelling is verified in the experimental results of all the specimens. Furthermore, the curvature of the experimental stress-strain curve during the tensile test is relatively small compared to the results of the $\mathrm{AB}$ model. The difference between the fitted and experimental curves of true stressstrain is discussed in the next section.

\section{DISCUSSION}

The relationship between true stress and true strain until the large-strain range was successfully evaluated using DIC. The relations obtained for each specimen with different cross-linkers, $\beta$, were fitted using the $\mathrm{AB}$ model and the respective material parameters. In the former part of this section, we discuss the effect of $\beta$ on the material parameters and attempt to relate them. Meanwhile, a comparison of the fitted and experimental results demonstrates that the $\mathrm{AB}$ model underestimates the decrease in the elasticity owing to the swelling, and overestimates the increase in the stress in the large-strain range. Therefore, we discuss the modification of the $\mathrm{AB}$ model to improve the accuracy of the mechanical model, in the later part of this section.

As shown in Table 3, $C_{R}$ and $N$ increases and decreases, respectively, as $\beta$ increases. To investigate the relationship between these parameters and $\beta$, we performed the additional tensile tests of the hydrogel specimens BIS1 and BIS15 presented in Table 4. The averaged response for each condition and the response fitted by the $\mathrm{AB}$ model are plotted in Fig. 5. The characteristics of the mechanical responses of the hydrogel are effectively reproduced by the $\mathrm{AB}$ model. The material parameters for the hydrogels with the different $\beta$ are plotted in Fig. 6. $C_{R}$ and the segment number for a chain $N$ nonlinearly increases and decreases, respectively, as $\beta$ increases. To reproduce the change in the $C_{R}$ for the hydrogels with the different $\beta$, we employ a simple power law with an exponent $\alpha$ as follows:

Table 4 Characteristics of hydrogel specimens

\begin{tabular}{ccccc}
\hline & BIS density $[\mathrm{mM}]$ & Swelling time & Volume increase ratio & Sample number \\
\hline BIS1-AP & 1 & As prepared & 1.0 & 3 \\
BIS1-SW & 1 & 24 hour & $4.29 \pm 0.04$ & 3 \\
BIS15-AP & 15 & As prepared & 1.0 & 3 \\
BIS15-SW & 15 & 24 hour & $2.27 \pm 0.01$ & 3 \\
\hline
\end{tabular}




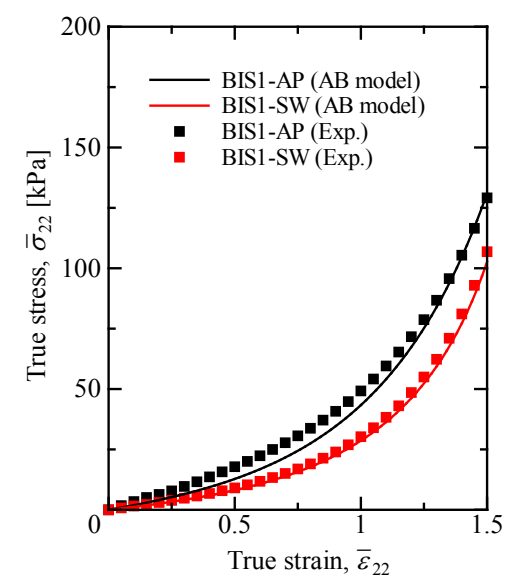

(a) BIS1

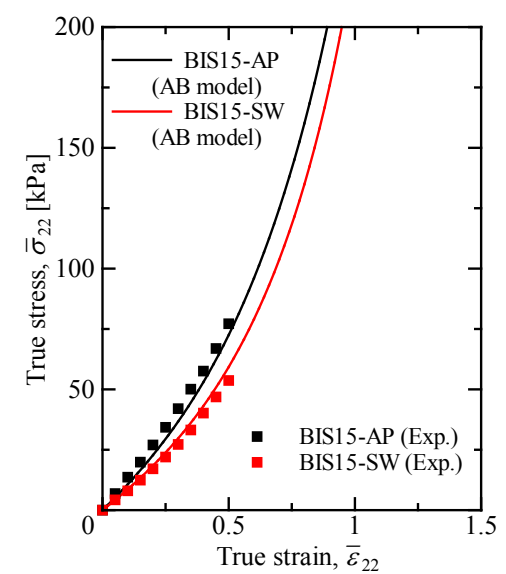

(b) BIS15

Fig. 5 Relationships between true stress and true strain fitted by AB model for BIS1 and 15.

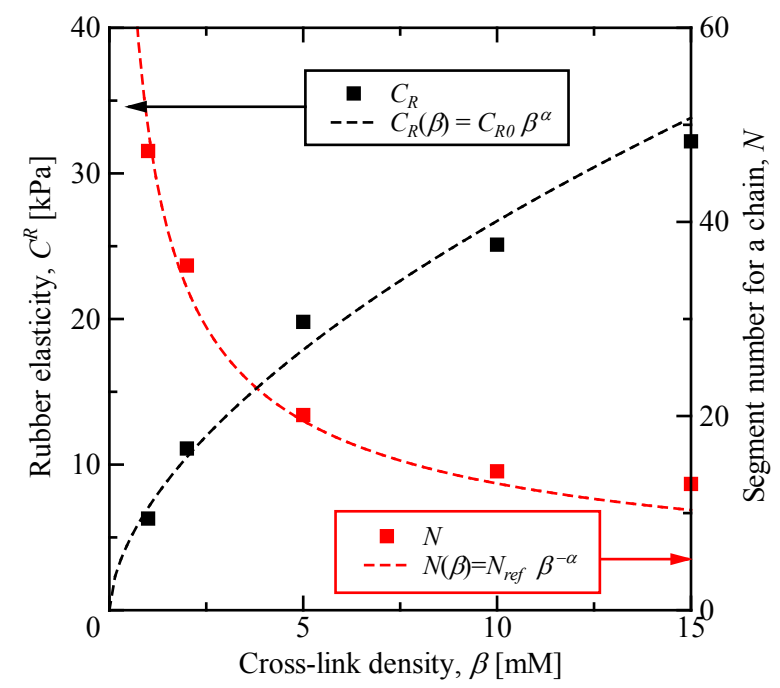

Fig. 6 Change in $C_{R}$ and $N$ for different $\beta$. The parameters for the fitted curves are

$$
C_{R 0}=7.03, \alpha=0.58 \text {, and } N_{0}=49.6
$$

$$
C_{R}(\beta)=C_{R 0} \beta^{\alpha},
$$

where $C_{R 0}$ is the rubber elasticity modulus for the hydrogel with $\beta=1$. This equation indicates that the cross-link density of the hydrogel increases nonlinearly with the cross-linker contents. Although a simple equation is employed to describe the $C_{R}$ for $\beta=1-15$, a more precise equation can be formulated based on the experiments of the hydrogels with the other values $\beta$.

The density of the total segment, which is proportional to the density of the monomer, can be expressed as a product of $n$ and $N$ [2]. As shown in Table 1, the contents of the monomer were maintained constant for all the specimens of the hydrogel prepared in this study. Therefore, $N(\beta)$ is expressed as 


$$
N(\beta)=\frac{n_{0}}{n(\beta)} N_{0}=\frac{C_{R 0}}{C_{R}(\beta)} N_{0}=N_{0} \beta^{-\alpha},
$$

where $N_{0}$ is the segment number for a chain in the hydrogel with $\beta=1$. Figure 6 also illustrates that $N(\beta)$ can be expressed by a negative power law using the $\alpha$ in Eq. (12). The material parameters of the hydrogels with the different $\beta$ are characterized by three parameters: $C_{R 0}, N_{0}$, and $\alpha$.

As mentioned in the previous section, the comparison of the fitted and experimental results demonstrates that the $\mathrm{AB}$ model underestimated the decrease in the elasticity owing to the swelling effect and overestimated the increase in the stress in the large-strain range. The reasons for these differences between the experiment and model are discussed here. The first important aspect is that the polymer chains of the hydrogels were in a solvent. The $\mathrm{AB}$ model was formulated for the free joint polymer network. The crosslinking process of the as-prepared specimen and the stretching of the single chain during the swelling and tensile test are likely to have been affected by the properties of the solvent. The likely effects are the change in the limit stretch of the chain crosslinked in the solvent, interactions with surrounding chains through the solvent, the viscous resistance of the solvent for the movement of the chain from the solvent, etc.

The second aspect is that the macroscopic response calculated by the $\mathrm{AB}$ model is based on the affine deformation, in which the deformation of the molecular chain is assumed to be in agreement with the global deformation. However, the non-affine deformation owing to the interaction of the neighboring molecular chains has been discussed for the gel [21, 22]. AB model overestimated the true stress in the large-strain range and underestimated the reduction in the elasticity of the swollen hydrogel. These inconsistencies indicate that the decrease in the rubber elastic modulus and increase in the critical stretch are likely to have occurred by the mechanical and swelling stretching of the polymer chain. Therefore, we attempted to establish the non-affine model wherein the development of the cross-link density was introduced in the $\mathrm{AB}$ model.

Generally, the cross-links of the polymer network are categorized into chemical and physical bonds. These correspond to the covalent bond and the bond by intermolecular force, respectively, wherein the physical bond is generally weaker than the chemical one [22]. In the proposed model, the non-affine deformation of the hydrogel was assumed to have been caused by the decrease in the physical bond because of the stretching of the hydrogel. This indicates the decrease in the rubber elasticity modulus and increase in $N$ during the swelling and the tensile test. A similar model has been adopted in the investigation of the mechanical responses for the glassy polymer [23] and rubber [24]. $C_{R}\left(\beta, \lambda_{c}\right)$ and $N\left(\beta, \lambda_{c}\right)$ are modified as

$$
\begin{aligned}
& C_{R}\left(\beta, \lambda_{c}\right)=C_{R}\left(\lambda_{c}\right) \beta^{\alpha}, \\
& N\left(\beta, \lambda_{c}\right)=N\left(\lambda_{c}\right) \beta^{-\alpha},
\end{aligned}
$$


where $\lambda_{c}$ is the chain stretch given by Eq. (9). $C_{R}$ and $N$ in Eq. (11) are replaced by $C_{R}\left(\beta, \lambda_{c}\right)$ in Eq. (14) and $N\left(\beta, \lambda_{c}\right)$ in Eq. (15), respectively. The cross-link density for the deformed hydrogel is expressed as

$$
n\left(\lambda_{c}\right)=n_{C 0}+n_{P}\left(\lambda_{c}\right)
$$

where $n_{C 0}$ is the cross-link density of the chemical bond, which is constant during deformation and $n_{P}\left(\lambda_{c}\right)$ is that for the physical bond, which changes depending on the degree of stretch of the chain. We assumed that the change in the physical bond with stretching, $d n_{P} / d \lambda_{c}$, is in proportional to the current physical bond density, $n_{P}$. The equation for such condition is expressed as

$$
n_{p}\left(\lambda_{c}\right)=A \exp \left\{r \lambda_{c}\right\}
$$

where $A$ and $r$ are material constants. By introducing the initial density of the physical bond, $\left(n_{p}(1)=n_{P 0}\right)$, we obtain

$$
n_{p}\left(\lambda_{c}\right)=n_{P 0} \exp \left\{r\left(\lambda_{c}-1\right)\right\}
$$

Therefore, rubber elasticity modulus, $C_{R}\left(\lambda_{c}\right)=n\left(\lambda_{c}\right) k_{B} T$, is expressed as

$$
C_{R}\left(\lambda_{c}\right)=C_{R C 0}+C_{R P 0} \exp \left\{r\left(\lambda_{c}-1\right)\right\},
$$

where $C_{R C 0}=n_{C 0} k_{B} T$ and $C_{R P 0}=n_{P 0} k_{B} T$ are the elastic moduli for polymer chains with chemical and physical bonds, respectively. $r$ corresponds to the decrease in the logarithm of the elastic modulus during a specified stretch. The number of segments for a chain is expressed as

$$
N\left(\lambda_{c}\right)=\frac{C_{R}(1)}{C_{R}\left(\lambda_{c}\right)} N_{0}=\frac{C_{R C 0}+C_{R P 0}}{C_{R C 0}+C_{R P 0} \exp \left\{r\left(\lambda_{c}-1\right)\right\}} N_{0} .
$$

In the proposed model, the mechanical behavior of as-prepared and swollen hydrogels with different $\beta$ are finally characterized by five parameters, namely, $C_{R C 0}, C_{R P 0}, N_{0}, \alpha$ and $r$.

The relationships between true stress and true strain obtained from the proposed model with the five parameters, which was fitted using the nonlinear least square method, are re-plotted in Fig. 7. For comparison, the results without introducing the development of physical bond are also plotted in the figure. It is observed that the proposed model is effectively describes the mechanical response of the as-prepared and swollen hydrogels with the different $\beta$ by five common parameters. Furthermore, the proposed model slightly improved the representation of the effect of swelling on the stress-strain curves. The fitted parameters employed in Fig. 7 are $C_{R C 0}=6.81 \mathrm{kPa}, C_{R P 0}=3.02 \mathrm{~Pa}$, 


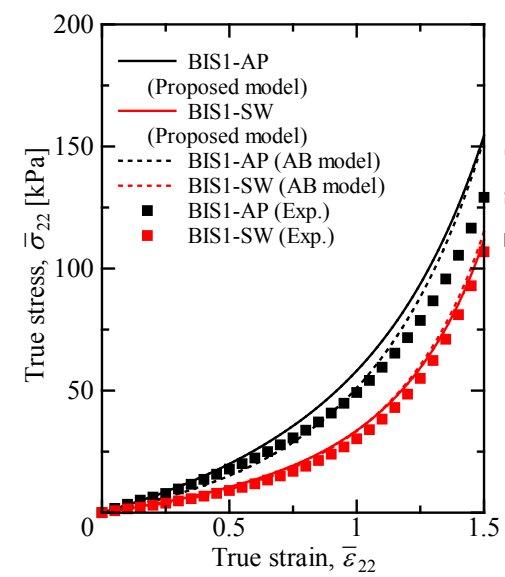

(a) BIS1

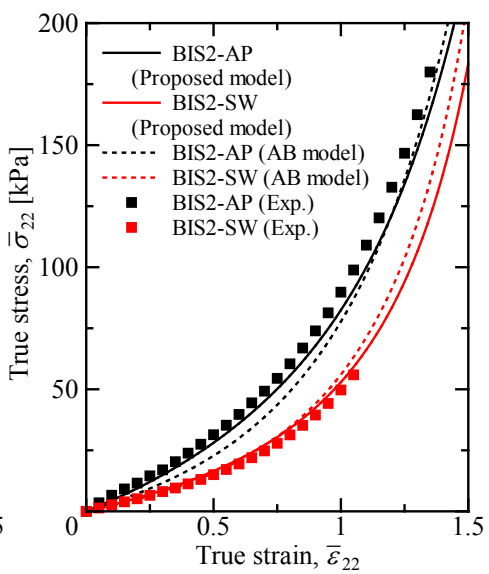

(b) BIS2

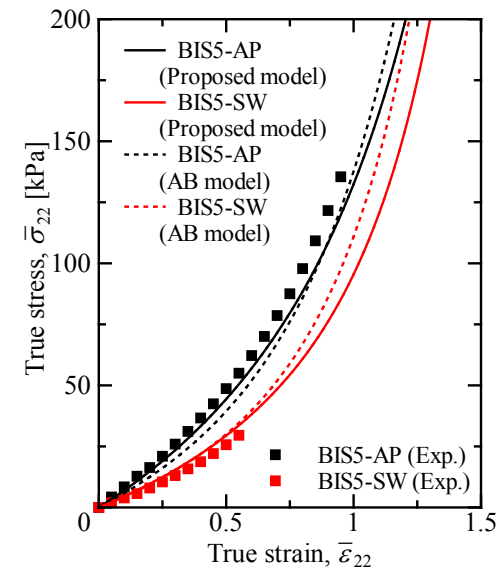

(c) BIS5

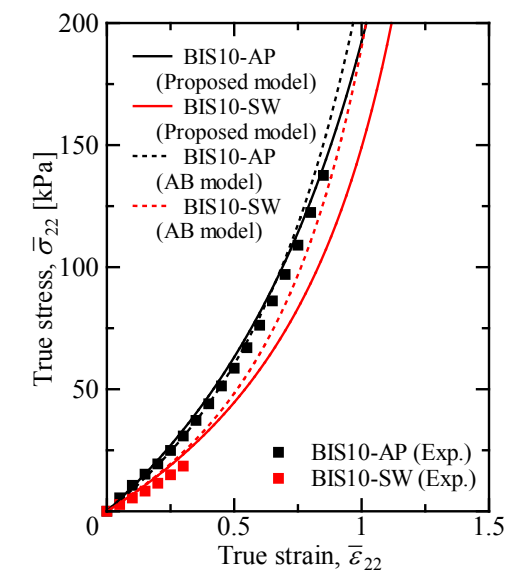

(d) BIS10

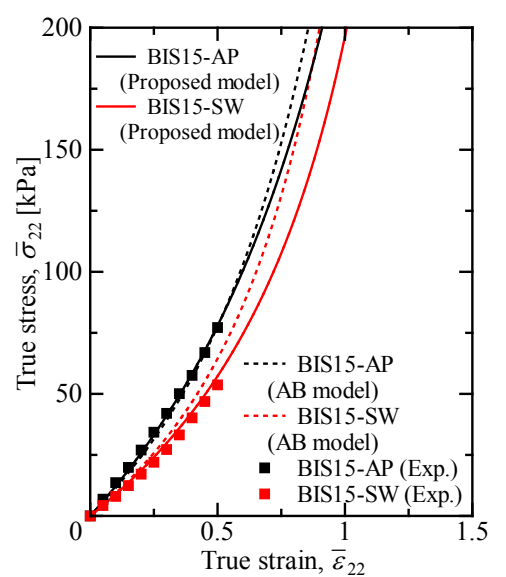

(e) BIS 15

Fig. 7 Relationships between true stress and true strain predicted by the proposed model.

$N_{0}=34.7 \quad \alpha=0.48$ and $r=1.5$.

In the present study, the mechanical behavior of the hydrogel was evaluated by DIC and was modeled by the molecular chain network theory. The advantage of DIC is not demonstrated under uniaxial and uniform deformation. However, DIC can be applied under more challenging mechanical conditions such as those encountered during the evaluation of a strain tensor under multiaxial stress state and the measurement of nonuniform deformation of a gel material with a complex shape. Moreover the experimentally evaluated deformation can be compared to those calculated by the computational simulation using the multiaxial constitutive equations. Mechanical tests under various conditions will enable further generalization of the theoretical model.

\section{CONCLUSION}

In this study, the effects of the cross-link density and swelling of polyacrylamide hydrogel on its true stress-strain relationship were evaluated using the uniaxial tensile test. The netDIC, which is an optical method for evaluating the two-dimensional strain field in the large-strain range by introducing a shape change in the subset using the displacement distribution on the network, was also 
employed. This was to evaluate the development of strain distribution on the specimen surface accurately and stably, from the small- to the large-strain range.

The true stress and strain were evaluated using the true strain obtained by DIC and the tensile loads applied on the specimen. The true stress-strain curve exhibited a response similar to the general rubber elasticity, which was accompanied by orientation hardening of the molecular chain in the largestrain range. The hydrogel specimens with larger cross-link density showed a higher initial elastic modulus and earlier orientation hardening. A decrease in the initial elastic modulus by an increase in the swelling was observed. It was also observed that the orientation hardening occurred at an earlier strain range in the swollen hydrogel. This result indicated that the solvent present in the polymer network caused the extension of the molecular chain, thereby resulting in the decrease in the extensibility.

The mechanical responses of the as-prepared and swollen hydrogels were fitted using the nonGaussian statistic Arruda-Boyce model (AB model). The comparison of the fitted and experimental results demonstrated that the $\mathrm{AB}$ model underestimated the decrease in the elasticity owing to the swelling effect, and overestimated the increase in the stress in the large-strain range. The AB model was suitably modified to yield accurate reproductions of the mechanical responses of the hydrogels with different cross-linker contents. The power law was employed to represent the change in the mechanical behavior of the hydrogel with respect to the cross-linker contents. Furthermore, the crosslinks of the hydrogel were categorized into chemical and physical bonds, and the number of physical bonds was assumed to decrease by the stretching of the hydrogel. The proposed model was found to successfully improve the accuracy of representation of the stress-strain curves of the hydrogels with different degrees of swelling and cross-link density by using five material parameters.

\section{CONFLICT OF INTEREST}

There are no conflicts of interest to declare.

\section{ACKNOWLEDGEMENT}

Authors gratefully acknowledge support from JSPS Grants-in-Aid for Scientific Research, Grant Numbers 16H04234.

\section{REFERENCE}

[1] P. J. Flory and J. Rehner, "Statistical mechanics of cross-linked polymer networks II. Swelling", Journal of Chemistry and Physics, Vol. 11, No. 11, (1943), pp. 521-526.

[2] M. C. Boyce and E. M. Arruda, "Swelling and mechanical stretching of elastomeric materials", Mathematics and Mechanics of Solids, Vol. 6, (2001), pp. 641-659.

[3] S. A. Chester and L. Anand, "A coupled theory of fluid permeation and large deformations for 
elastomeric materials", Journal of the Mechanics and Physics of Solids, Vol. 58, No. 11, (2010), pp. 1879-1906.

[4] D. Okumura, A. Kondo and N. Ohno, "Using two scaling exponents to describe the mechanical properties of swollen elastomers" Journal of Mechanics and Physics of Solids, Vol. 90, (2016), pp. 61-76.

[5] D. Okumura, M. Mizutani, H. Tanaka and M. Uchida, "Effects of two scaling exponents on biaxial deformation and mass transport of swollen elastomers", International Journal of Mechanical Sciences, Vol. 3, (2018), pp. 135-140.

[6] D. Okumura and S. A. Chester, "Ultimate swelling described by limiting chain extensibility of swollen elastomers", International Journal of Mechanical Sciences, Vol. 144, (2018), pp. 531539.

[7] A. D. Drozdov and J. deC. Christiansen, "Constitutive equations in finite elasticity of swollen elastomers", International Journal of Solid and Structures, Vol. 50, (2013), pp. 1494-1504.

[8] V. Normand, D. L. Lootens, E. Amici, K. P. Plucknett and P. Aymard, "New insight into agarose gel mechanical properties", Biomacromolecules, Vol. 1, No. 4, (2000), pp. 730-738.

[9] J. A. Stammen, S. Williams. D. N. Ku and R. E. Guldberg, "Mechanical properties of a novel PVA hydrogel in shear and unconfined compression”, Biomaterials, Vol. 22, No. 8, (2001), pp. 799-806

[10] W. Xue, S. Champ and M. B. Huglin, "Network and swelling parameters of chemically crosslinked thermoreversible hydrogels", Polymer, Vol. 42, (2001), pp. 3665-3669.

[11] D. Calvet, J. Y. Wong, and S. Giasson, "Rheological monitoring of polyacrylamide gelation: Importance of cross-link density and temperature", Macromolecules, Vol. 37 (2004), pp. 7762 7771.

[12] R. Mishima, A. Nakao, S. Sakurai and K. Urayama, "Peculiar extensibility of swollen statistical hydrogels with structural nanoheterogeneities", Polymer, Vol. 115, (2017), pp. 28-36.

[13] H. J. Kwon, A. D. Rogalsky, C. Kovalchick, G. Ravichandran, "Application of digital image correlation method to biogel", Polymer Engineering and Science, Vol. 50, No. 8, (2010), pp. 1585-1593.

[14] G. Subhash, Q. Liu, D. F. Moore, P. G. Ifju and M. A. Haile, "Concentration dependence of tensile behavior in agarose gel using digital image correlation", Experimental Mechanics, Vol. 51, (2011), pp. $255-262$.

[15] F. Chen, D. J. Kang and J. H. Park, "New measurement method of Poisson's ratio of PVA hydrogels using an optical flow analysis for a digital imaging system", Measurement Science and Technology, Vol. 24, (2013), 055602 (8pp).

[16] K. Suzuki, M. Uchida, Y. Kaneko, D. Okumura, H. Tanaka and S. Ida, "Evaluation of deformation behavior of hydrogel on uniaxial tensile test using digital image correlation", Proceedings of the 
12th International Symposium on Advanced Science and Technology in Experimental Mechanics, (2017), Paper No. 059, 5pp.

[17] M. Uchida and N. Tada, "Sequential evaluation of continuous deformation field of semicrystalline polymers during tensile deformation accompanied by neck propagation", International Journal of Plasticity, Vol. 27, No. 12, (2011), pp. 2085-2102.

[18] M. Uchida, T. Ueno, T. Abe and Y. Kaneko, "Quantitative evaluation of the development of stress and strain fields using digital image correlation and finite element methods", Advanced Experimental Mechanics, Vol. 2 (2017), pp. 76-81.

[19] M. Uchida, A. Taniguchi, Y. Kaneko, "Estimation of slip deformation of polycrystalline pure copper using digital image correlation method and crystalline plasticity theory", Advanced Experimental Mechanics, Vol. 3 (2018), pp. 135-140.

[20] E. M. Arruda and M. C. Boyce, "A three-dimensional constitutive model for the large stretch behavior of rubber elastic materials", Journal of the Mechanics and Physics of Solids, Vol. 41, No. 2 (1993), pp. 389-412.

[21] R. Michael and P. Sergei Panyukov, "Nonaffine deformation and elasticity of polymer networks", Macromolecules, Vol. 30, No. 25 (1997), pp 8036-8044.

[22] W. Qi, B. Anindita A. J. Paul and A. G. Yodh, "Non-affine deformations in polymer hydrogels", Soft Matter, Vol. 8, No. 31 (2012), pp. 8039-8049.

[23] Y. Tomita and S. Tanaka, "Prediction of deformation behavior of glassy polymers based on molecular chain network model”, International Journal of Solids and Structures, Vol. 32, No. 23 (1995), pp. 3423-3434.

[24] Y. Tomita, W. Lu, M. Naito and Y. Furutani, "Numerical evaluation of micro- to macroscopic mechanical behavior of carbon-black-filled rubber", International Journal of Mechanical Sciences, Vol. 48, No. 2 (2006), pp. 108-116. 